\section{Ontogenèse des cellules germinales primordiales}

Nadejda Machev, Guy Fuhrmann, Stéphane Viville
> Chez les animaux à reproduction sexuée, les gamètes proviennent d'une petite population cellulaire apparaissant très tôt au cours du développement embryonnaire, les cellules germinales primordiales ( $P G C)$. Ces cellules représentent une population cellulaire clé responsable de la survie et de l'évolution des espèces. En effet, la production de gamètes permet la fécondation et donc l'établissement de la génération suivante. Chez les mammifères, l'étude des PGC est restée, jusqu'à peu, très confidentielle. Elles ont récemment acquis un nouvel intérêt dans la mesure où il est possible d'en dériver des cellules pluripotentes, les cellules germinales embryonnaires $(\varepsilon G C)$, dont les caractéristiques sont très proches de celles des cellules souches embryonnaires (ESC). <

Les cellules germinales embryonnaires ( $E G C$ ) sont capables de se différencier en une grande variété de types cellulaires in vitro comme in vivo [1-5]. De ce fait, certains auteurs considèrent qu'elles ont un potentiel en thérapie de remplacement aussi important que celui des cellules souches embryonnaires (ESC) [6]. Cependant, si cette pluripotence semble bien établie pour les lignées murines [2, 3], elle doit être étudiée plus en détail chez l'homme. Nous nous proposons dans cet article de présenter l'ontogenèse des cellules germinales primordiales, en particulier dans le modèle murin.

\section{L’ontogenèse des PGC, un phénomène conservé?}

La détermination des PGC fait intervenir des mécanismes très différents selon les espèces [7]. Chez la drosophile, le nématode et le xénope, des déterminants cytoplasmiques d'origine maternelle, déjà présents dans l'ovocyte (granules polaires chez la drosophile ou granules $P$ chez le nématode), ont un rôle essentiel. Chez les mammifères, la présence de précurseurs communs aux PGC et aux cellules somatiques n'est pas en

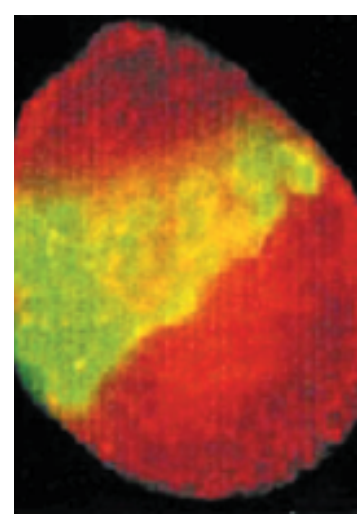

Institut de Génétique et de biologie moléculaire et cellulaire, CNRS/Inserm/ULP, BP 10142, 1, rue Laurent Fries, 67404 IIlkirch Cedex, CU de Strasbourg, France et Service de Biologie de la reproduction, SIHCUSCMCO, CHU de Strasbourg, 19, rue Louis Pasteur, BP 120, 67303 Schiltigheim Cedex,

faveur de l'existence France.

de déterminants cyto- viville@igbmc.u-strasbg.fr plasmiques.

Cependant, certaines caractéristiques sont conservées dans toutes les espèces étudiées. Ainsi, on observe toujours une séparation spatiotemporelle de la différenciation des PGC et des gonades, et une nécessité pour les PGC de migrer jusqu'aux ébauches gonadiques [8] : chez les mammifères, les PGC sont déterminées au moment de la gastrulation (7,2 jours de développement chez la souris et 22-24 jours de développement chez I'homme), tandis que les ébauches gonadiques apparaissent plus tardivement (10,5 jours chez la souris et 42-44 jours chez l'homme). Dans pratiquement toutes les espèces, les PGC vont devoir migrer pour coloniser les gonades [8] : cette migration s'effectue selon deux modes, une migration passive contemporaine des mouvements morphogénétiques à l'origine de la forme du corps, et une migration active. Si la détermination des PGC chez les insectes et les mammifères fait intervenir des mécanismes très différents, les phénomènes de migration et de prolifération pourraient, en revanche, partager certains mécanismes moléculaires [9].

\section{Origine des PGC}

K.A. Lawson et W.J. Hage ont démontré, chez la souris, que les précurseurs des PGC provenaient de l'épiblaste, et plus précisément de l'épiblaste proximal proche de 
l'ectoderme extra-embryonnaire (Figure 1). À 6,0 jours de développement, ces précurseurs, qui donnent aussi naissance à des dérivés du mésoderme extra-embryonnaire [10], sont présents sur tout le pourtour de cet épiblaste proximal. Au cours de la gastrulation (à partir de 6,5 jours), ils vont être exclus de la future région antérieure de cet épiblaste et migrer hors de l'épiblaste par la partie postérieure de la ligne primitive pour se localiser dans une région extra-embryonnaire à la base de l'allantoïde. C'est à partir de cette étape du développement - 7,2 jours - que le potentiel de ces progéniteurs se restreint à la lignée des PGC, une fois leur localisation extra-embryonnaire réalisée. À ce stade, et durant toute leur migration, les PGC sont identifiées par l'expression de certains gènes tels que celui codant pour la phosphatase alcaline, SSEA-1, F9, $\varepsilon M A-1,0 c t 4$ et Stella $[11,14]$, marqueurs également caractéristiques des cellules souches embryonnaires.

Des expériences de transplantation d'épiblaste de 6,5 jours ont confirmé ces résultats et démontré la plasticité de l'épiblaste à ce stade du développement embryonnaire. Ainsi, une transplantation d'un épiblaste proximal en position distale ne donnera pas de PGC, alors que la greffe d'un épiblaste distal en position proximale permet d'obtenir des PGC (Figure 1). Cette étude suggère l'importance des signaux provenant de l'environnement dans le déterminisme des PGC [15], et en particulier de l'importance de la proximité de l'ectoderme extraembryonnaire. De même, la mise en culture d'un épiblaste proximal de 6,0 jours donne naissance à des PGC, alors qu'un épiblaste distal ne donne jamais naissance à des PGC, sauf s'il est placé en présence d'ectoderme extra-embryonnaire. Ces résultats établissent clairement le rôle inducteur de l'ectoderme extra-embryonnaire dans l'émergence des PGC [16].

\section{Déterminisme et différenciation des PGC}

Parmi les voies de signalisation contrôlant le déterminisme et la différenciation des PGC, il semble que celle des membres de la famille TGF $\beta$ joue un rôle primordial. Les membres de cette grande famille contiennent d'une part le TGF $\beta$, l'activine et Nodal et, d'autre part, les BMP (bone morphogenic proteins), tous facteurs connus pour être sécrétés et impliqués dans nombre de phénomènes de différenciation au cours de l'embryogenèse. Concernant les $P G C$, trois membres de la sous-famille des BMP semblent impliqués dans leur spécifica- tion et leur différenciation. L'étude des souris mutantes pour Bmp4, Bmp2, Bpm8b et pour deux de leurs effecteurs intracellulaires, Smadl et Smad5, a conduit à l'élaboration d'un modèle impliquant séquentiellement ces trois signaux (Figure2) : une première exposition à Bmp4, synthétisée par l'ectoderme extra-embryonnaire, confèrerait à l'épiblaste proximal une compétence à se différencier soit en PGC, soit en allantoïde. La restriction dans la lignée des PGC serait contrôlée par un deuxième signal provenant conjointement de l'ectoderme extra-embryonnaire via Bmp4 et Bmp8b et de I'endoderme extra-embryonnaire via Bmp2. Par la suite, la survie et la localisation des PGC seraient sous le contrôle de Bmp4 synthétisée par le mésoderme extraembryonnaire [17].

L'expression de Bmp4 est limitée à l'ectoderme extraembryonnaire proximal de 6,0 jours à 6,5 jours de développement, puis s'étend lors de la gastrulation au mésoderme extra-embryonnaire. Les embryons porteurs d'une invalidation homozygote du gène bmp 4 sont totalement dépourvus de PGC, et une réduction de $50 \%$ de leur nombre est observée chez les hétérozygotes [18]. La protéine Bmp4, exprimée par l'ectoderme extra-embryonnaire, induirait la spécification des PGC,

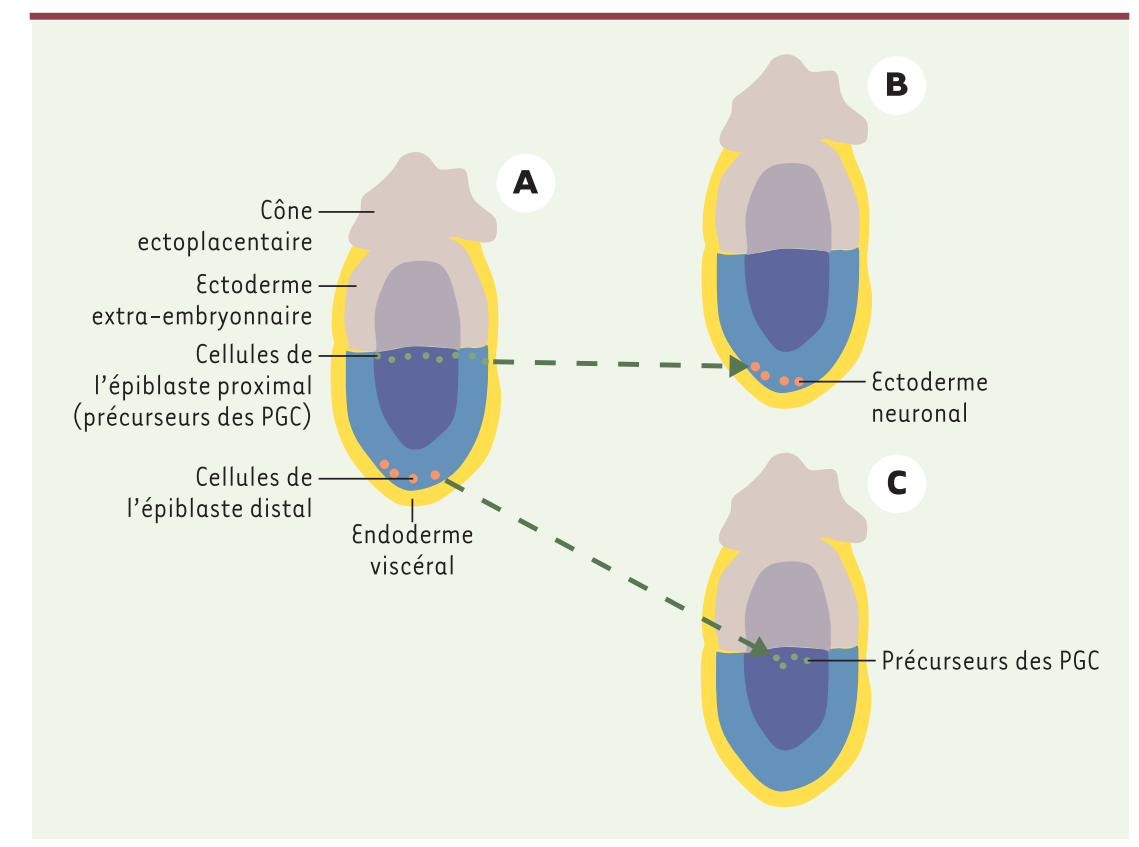

Figure 1. Localisation des PGC chez l'embryon de souris à 6,0 jours de développement. A. Les précurseurs des cellules germinales primordiales (PGC) (en vert) sont retrouvés dans la région proximale de l'épiblaste, juste à la limite épiblaste/ectoderme extra-embryonnaire. B. La transplantation de ces cellules dans une région distale de l'épiblaste aboutit à la formation de l'ectoderme neuronal (en rouge), prouvant ainsi l'absence de déterminisme de ces cellules à ce stade du développement. $C$. Inversement, la transplantation de cellules provenant de la région distale de l'épiblaste (en rouge) en région proximale permet l'obtention de PGC (en vert), prouvant ainsi l'importance de l'environnement dans la différenciation des PGC. 
tandis que la protéine Bmp4 du mésoderme extraembryonnaire assurerait leur localisation et leur survie $[18,19]$.

L'expression de Bmp8b est limitée, entre 6,0 jours et 7,5 jours, à l'ectoderme extra-embryonnaire, et l'absence de Bmp8b entraîne une diminution du nombre, voire une absence totale des PGC. Bmp8b interviendrait, comme Bmp4, dans la détermination des PGC, mais pas dans leur prolifération ni dans leur survie. $\varepsilon$ n effet, chez certains embryons homozygotes dépourvus de Bmp8b, lorsque des PGC sont retrouvées, elles migrent et prolifèrent de façon comparable à ce qui est observé chez les embryons hétérozygotes [20, 21].

Des expériences de culture d'épiblastes distaux ont établi que Bmp4 induirait, très tôt au cours du développement ( 6,0 jours), une compétence au niveau de cet épiblaste à donner des PGC [22]. De plus, Bmp4 et Bmp8b agiraient, sous forme d'homodimères et non d'hétérodimères, directement sur l'épiblaste par une action synergique via deux voies de signalisation différentes qui demeurent inconnues [22].

L'étude de souris mutantes pour Bmp2, qui est exprimée dans la région postérieure de l'endoderme viscéral, a montré une réduction du nombre de PGC. Tout comme pour Bmp4, cette réduction est dépendante de la dose puisqu'une réduction est déjà observée chez les embryons hétérozygotes. Bmp2 interviendrait seulement dans la différenciation des PGC.

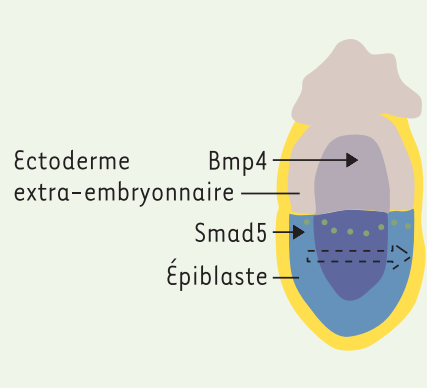

$6,0 \mathrm{j}$

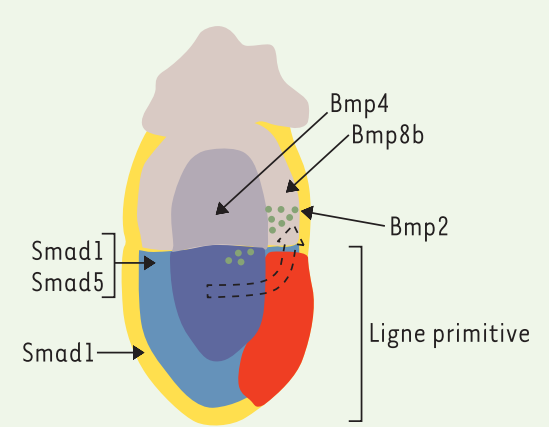

$6,5 j$
L'étude d'embryons double hétérozygotes, soit pour Bmp2 et Bmp4, soit pour Bmp2 et Bmp8b, a permis d'établir que Bmp4 et Bmp2 agissaient de façon additive sur la différenciation des PGC, alors que ce n'est pas le cas pour Bmp2 et Bmp8b [23]. Les auteurs de cette étude proposent, au vu de la forte similitude entre Bmp2 et Bmp4, que ces deux facteurs interviendraient via la même voie de signalisation.

Actuellement, les récepteurs exprimés par les PGC et impliqués dans ces voies de signalisation ne sont pas connus. En revanche, les facteurs intracytoplasmiques commencent à êtres identifiés. Ainsi, les souris mutantes pour Smadl ou Smad5 présentent une forte réduction, voir une absence totale de PGC. II reste à définir à quel niveau exact Smadl et Smad 5 interviennent. Le profil d'expression de Smadl, exprimé à 6,5jours au niveau de l'endoderme viscéral et dans une moindre mesure au niveau de l'épiblaste, suggère qu'il serait impliqué dans la prolifération/survie des PGC [24]. En revanche, Smad5, exprimé à 6,5 jours par l'épiblaste, serait l'effecteur cytoplasmique de Bmp4 et éventuellement de Bmp8b (Figure 2). En effet, les mutants Smad5 présentent une réduction du nombre de $P G C$ et une localisation anormale, ce qui est aussi observé chez les mutants Bmp8b [25].

Des travaux récents ont démontré la possibilité de différencier des cellules souches embryonnaires (ES) en PGC, et ultérieurement en ovocytes ou spermatocytes [26-28]. Les conditions expérimentales semblent déterminantes pour favoriser l'une ou l'autre gamétogenèse. Notamment, le rôle de différents facteurs trophiques ajoutés dans le milieu de culture ou synthétisés par les cellules souches en voie de différenciation a été clairement spécifié. Le rôle du microenvironnement dans lequel baignent les cellules souches embryonnaires est capital pour une orientation sélective vers l'ovogenèse ou la spermatogenèse. Notons que la différenciation ovocytaire observée dans la culture de cellules $E S$ se poursuit jusqu'à la formation de follicules matures producteurs d'hormones œstrogéniques. De plus, probablement par un phénomène parthénogénétique, l'apparition de structures blastocytaires a pu être observée [26].

\section{Migration des PGC}

Figure 2. Modèle à trois signaux expliquant l'émergence des PGC à partir de l'épiblaste proximal. À 6,0 jours de développement, le facteur Bmp4 (bone morphogenic protein) synthétisé par l'ectoderme extra-embryonnaire confèrerait une compétence à l'épiblaste à se différencier en PGC. Ce signal ferait intervenir au niveau de l'épiblaste proximal Smad5. Un deuxième signal plus tardif, à 6,5 jours, via Bmp4, Bmp8b et Bmp2, serait responsable de la différenciation des PGC. L'expression de Smadl à 6,5 jours, au niveau de l'endoderme viscéral et, dans une moindre mesure, au niveau de l'épiblaste, suggère qu'il serait impliqué dans la prolifération et la survie des PGC. Smad5, exprimé à 6,5 jours dans l'épiblaste, serait effecteur cytoplasmique de Bmp4, et éventuellement de Bmp8b. Au cours de la gastrulation, les précurseurs des PGC présents sur le pourtour de l'épiblaste proximal migrent hors de l'épiblaste pour rejoindre une région extraembryonnaire à la base de la future allantoïde (flèches noires pointillées).
Les précurseurs des PGC migrent à la base de l'allantoïde en traversant la partie postérieure de la ligne primitive. De là, les PGC se dispersent pour rejoindre les structures adjacentes, comme l'allantoïde, l'endoderme primitif ou l'endoderme définitif. Actuellement, peu de marqueurs spécifiques sont connus. Aucun marqueur spécifique des différentes phases de migration des PGC n'a encore été décrit. 
Deux modes de migration, l'un passif, l'autre actif, vont permettre aux PGC de rejoindre les crêtes génitales (Figure 3). La formation de l'endoderme de la paroi de l'intestin postérieur à partir de l'endoderme viscéral conduit, de façon passive, à l'intégration des PGC dans ce tissu [29]. Elles vont ensuite activement migrer pour quitter l'intestin, traverser le mésentère dorsal et rejoindre les ébauches gonadiques. Celles-ci se forment de chaque côté de la ligne médiane, entre le mésonéphros et le mésentère dorsal, du fait de la prolifération de l'épithélium cœlomique et d'une condensation du mésenchyme sous-jacent. II semble que les deux modes de migration se fassent simultanément. En effet, les PGC possèdent une morphologie de cellules migratrices (présence de pseudopodes) dès 7,5 jours de développement [29].

Les premières cellules arrivent dès 9,5 jours sur le lieu des futures gonades. Ces résultats permettent d'émettre l'hypothèse selon laquelle les premières cellules «pionnières» guideraient les PGC suivantes vers leur destination grâce aux contacts qu'elles conservent entre elles via leurs pseudopodes [30]. Cette migration serait favorisée par un effet chimio-attractant des gonades à 10,5 jours [31]. L'étude in vitro des propriétés d'adhérence des PGC à la $\beta$-caténine, au collagène IV et à la laminine a montré que l'affinité des PGC à ces trois molécules de la matrice extracellulaire variait au cours de leur migration. Une fois dans les gonades, les PGC présentent notamment une augmentation de leurs propriétés d'adhérence à la laminine, qui y est fortement exprimée [32]. Différentes molécules d'adhérence sont exprimées par les PGC ( $\varepsilon$ cadhérine, $\mathrm{N}$-cadhérine, $\mathrm{P}$-cadhérine, $\beta$-caténine, $\varepsilon p$ CAM et les intégrines $\alpha 3, \alpha 6, \alpha V, \beta 1$ et $\beta 3)[33,34]$ ou par les cellules somatiques avec lesquelles elles interagissent (collagène IV, fibronectine et laminine) [32]. Cependant il n'a pu être attribué un rôle, dans cette migration, qu'à la sous-unité $\beta 1$ des intégrines, sans que l'on connaisse la sous-unité $\alpha$ avec laquelle elle s'hétérodimérise [33].

D'autres facteurs sont impliqués dans la survie, la prolifération et la migration des PGC. C'est le cas, par exemple, des mutations dominant white spoting $(W)$ et le locus Steel [12]. Ces locus codent, respectivement, pour c-kit et son ligand le facteur Steel (SF) [35], mais leur rôle n'est pas encore élucidé.

\section{Conclusions}

Le déterminisme des PGC fait intervenir des mécanismes très différents selon les espèces. Ainsi, chez la drosophile, le xénope ou le nématode, ce déterminisme dépend de facteurs cytoplasmiques déjà présents dans l'ovocyte et donc d'origine maternelle. Les cellules héritant de ces facteurs donneront des PGC et uniquement des PGC. Chez les mammifères, aucun facteur cytoplasmique d'origine maternelle n'a été mis en évidence. Au contraire, il a été clairement établi que les précurseurs des PGC pouvaient aussi contribuer à la formation d'autres tissus. En revanche, une fois déterminées - et quelles que soient les espèces étudiées - leur ontogenèse suit un schéma très proche. Ainsi, il est toujours observé une séparation spatiotemporelle de la formation des PGC et des ébauches gonadiques. De plus, le premier site où elles sont observées, en tant que population cellulaire déterminée, est toujours extraembryonnaire ou à la marge de l'embryon, puis il y a une migration de ces cellules vers les ébauches gonadiques. II semble que les PGC soient temporairement exclues de l'embryon à un moment où se met en place la différenciation somatique. Certains auteurs $[7,36]$ pensent que cela leur permet d'échapper au processus de spécification des tissus somatiques. C'est aussi l'hypothèse avancée pour expliquer l'inhibition de la transcription observée dans les PGC de drosophile et de nématode, rendant ces cellules incapables de répondre aux diffé-

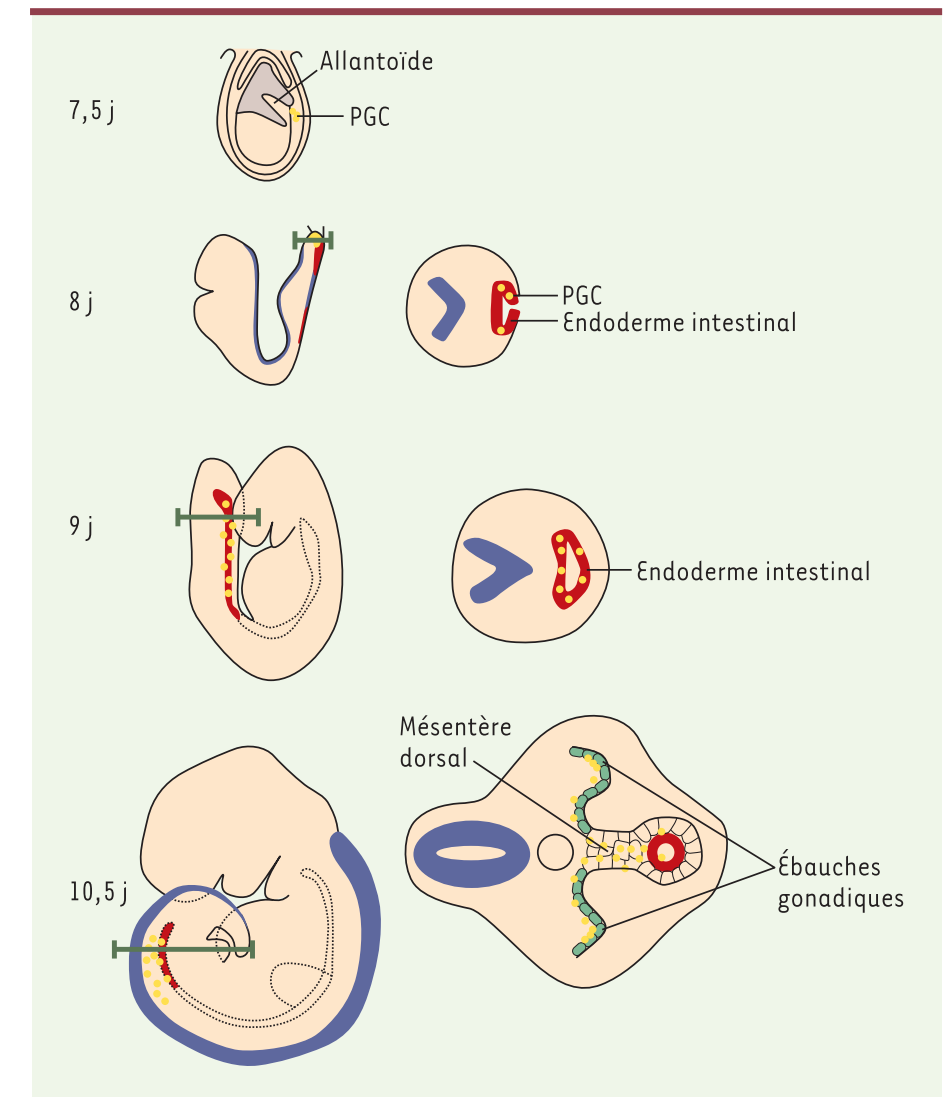

Figure 3. Migration des PGC au cours du développement embryonnaire chez la souris. À 7,5 jours, les PGC sont retrouvées à la base de l'allantoïde, dans l'endoderme définitif. Entre les $8^{\mathrm{e}}$ et $9^{\mathrm{e}}$ jours, du fait de mouvements morphogénétiques, elles vont être incorporées dans l'endoderme intestinal. De là, elles vont migrer activement pour rejoindre les ébauches gonadiques via le mésentère dorsal. 
rents signaux responsables des phénomènes de différenciation. Les travaux décrits ici, ne représentent que les quelques données que nous possédons de la biologie des PGC chez les mammifères. Des études plus approfondies sont donc nécessaires, cela afin de mieux appréhender les problèmes d'infertilité chez I'homme. De plus, les PGC ont potentiellement un avenir en thérapie de remplacement, dans la mesure où il semble qu'elles peuvent être à l'origine de lignées cellulaires pluripotentes, au même titre que les ESC. $\diamond$

\section{SUMMARY}

\section{Ontogenesis of primordial germ cells}

In sexually reproducing animals all gametes of either sex arise from primordial germ cells (PGC). PGC represent a small cell population, appearing early during embryo development. They represent a key cell population responsible for the survival and the evolution of a species. Indeed, the production of gametes will assure fertilisation and therefore the establishment of the next generation. Until recently only few laboratories were working on PGC biology. A new interest emerged since these cells have the ability to function as pluripotent stem cells when established as cell lines. Indeed, like embryonic stem cells (ESC), embryonic germ cells ( $(G C)$ are able to differentiate in a wide variety of tissues. In vivo, $\varepsilon G C$ are able, after injection into a host blastocyst cavity to colonise the inner cell mass and to participate in embryonic development. In vitro studies in human and mouse have also shown their capacity to differentiate into a large variety of cell types allowing the study of processes involved in cardiomyocyte, haematopoietic, neuronal and myogenic differentiation pathways. We present here the last updates of PGC ontogeny focusing mainly on the murine model. $\diamond$

\section{RÉFÉRENCES}

1. Kerr DA, Llado J, Shamblott MJ, et al. Human embryonic germ cell derivatives facilitate motor recovery of rats with diffuse motor neuron injury. J Neurosci 2003 ; $23: 5131-40$.

2. Durcova-Hills G, Wianny F, Merriman J, et al. Developmental fate of embryonic germ cells (EGCs), in vivo and in vitro. Differentiation $2003 ; 71: 135-41$.

3. Labosky PA, Barlow DP, Hogan BL. Mouse embryonic germ ( $\varepsilon G)$ cell lines : Transmission through the germline and differences in the methylation imprint of insulin-like growth factor 2 receptor (Igf2r) gene compared with embryonic stem (ES) cell lines. Development $1994 ; 120: 3197-204$.

4. Shamblott MJ, Axelman J, Wang $S$, et al. Derivation of pluripotent stem cells from cultured human primordial germ cells. Proc Natl Acad Sci USA 1998 ; 95 : 13726-31.

5. Shamblott MJ, Axelman J, Littlefield JW, et al. Human embryonic germ cell derivatives express a broad range of developmentally distinct markers and proliferate extensively in vitro. Proc Natl Acad Sci USA $2001 ; 98: 113-8$.

6. Gearhart J. New potential for human embryonic stem cells. Science $1998 ; 282$ : 1061-2.

7. Dixon KE. Evolutionary aspects of primordial germ cell formation. Ciba Found Symp 1994 ; 182: 92-110; discussion 110-20.

8. Starz-Gaiano M, Lehmann R. Moving towards the next generation. Mech Dev 2001 ; $105: 5-18$

9. Noce T, Okamoto-Ito S, Tsunekawa N. Vasa homolog genes in mammalian germ cell development. Cell Struct Funct $2001 ; 26: 131-6$.
10. Lawson KA, Hage WJ. Clonal analysis of the origin of primordial germ cells in the mouse. Ciba Found Symp 1994 ; 182 : 68-84; discussion 84-91.

11. Saitou M, Barton SC, Surani MA. A molecular programme for the specification of germ cell fate in mice. Nature $2002 ; 418: 293-300$.

12. Mintz B, Russell ES. Gene-induced embryological modifications of primordial germ cells in the mouse. J Exp Zool 1957 ; $134: 207-37$.

13. Ginsburg M, Snow MH, McLaren A. Primordial germ cells in the mouse embryo during gastrulation. Development $1990 ; 110: 521-8$.

14. Hahnel $A, \varepsilon d d y$. Cell surface markers of mouse primordial germ cells defined by two monoclonal antibodies. Gamete Res $1986 ; 15: 25-34$.

15. Tam PP, Zhou SX. The allocation of epiblast cells to ectodermal and germ-line lineages is influenced by the position of the cells in the gastrulating mouse embryo. Dev Biol $1996 ; 178$ : 124-32.

16. Yoshimizu T, Obinata M, Matsui Y. Stage-specific tissue and cell interactions play key roles in mouse germ cell specification. Development 2001 ; $128: 481-90$.

17. McLaren A. Signaling for germ cells. Genes Dev 1999 ; 13 : 373-6.

18. Lawson KA, Dunn NR, Roelen BA, et al. Bmp4 is required for the generation of primordial germ cells in the mouse embryo. Genes Dev 1999; $13: 424-36$.

19. Fujiwara T, Dunn NR, Hogan BL. Bone morphogenetic protein 4 in the extraembryonic mesoderm is required for allantois development and the localization and survival of primordial germ cells in the mouse. Proc Natl Acad Sci USA $2001 ; 98$ : 13739-44.

20. Zhao GQ, Deng K, Labosky PA, et al. The gene encoding bone morphogenetic protein $8 \mathrm{~B}$ is required for the initiation and maintenance of spermatogenesis in the mouse. Genes Dev 1996 ; $10: 1657-69$

21. Ying $Y$, Liu XM, Marble A, et al. Requirement of Bmp8b for the generation of primordial germ cells in the mouse. Mol Endocrinol $2000 ; 14: 1053-63$.

22. Ying $Y$, Qi $X$, Zhao GQ. Induction of primordial germ cells from murine epiblasts by synergistic action of BMP4 and BMP8B signaling pathways. Proc Natl Acad Sci USA $2001 ; 98: 7858-62$.

23. Ying $Y$, Zhao GP. Cooperation of endoderm-derived BMP2 and extraembryonic ectoderm-derived BMP4 in primordial germ cell generation in the mouse. Dev Biol $2001 ; 232: 484-92$.

24. Tremblay KD, Dunn NR, Robertson $\varepsilon$ J. Mouse embryos lacking Smadl signals display defects in extra-embryonic tissues and germ cell formation. Development 2001 ; $128: 3609-21$.

25. Chang H, Matzuk MM. Smad5 is required for mouse primordial germ cell development. Mech Dev 2001104 : 61-7.

26. Hubner K, Fuhrmann G, Christenson LK, et al. Derivation of oocytes from mouse embryonic stem cells. Science $2003 ; 300: 1251-6$.

27. Toyooka Y, Tsunekawa N, Akasu R, Noce T. Embryonic stem cells can form germ cells in vitro. Proc Natl Acad Sci USA 2003 ; 100 : 11457-62.

28. Geijsen N, Horoschak M, Kim K, et al. Derivation of embryonic germ cells and male gametes from embryonic stem cells. Nature 2004 ; 427 : 148-54.

29. Anderson R, Copeland TK, Scholer $\mathrm{H}$, et al. The onset of germ cell migration in the mouse embryo. Mech Dev $2000 ; 91: 61-8$.

30. Gomperts M, Garcia-Castro M, Wylie C, Heasman J. Interactions between primordial germ cells play a role in their migration in mouse embryos. Development 1994 ; $120: 135-41$.

31. Godin I, Wylie C, Heasman J. Genital ridges exert long-range effects on mouse primordial germ cell numbers and direction of migration in culture. Development $1990 ; 108: 357-63$.

32. Garcia-Castro MI, Anderson R, Heasman J, Wylie C. Interactions between germ cells and extracellular matrix glycoproteins during migration and gonad assembly in the mouse embryo. J Cell Biol 1997 ; $138: 471-80$.

33. Anderson R, Fassler R, Georges-Labouesse $\varepsilon$, et al. Mouse primordial germ cells lacking betal integrins enter the germline but fail to migrate normally to the gonads. Development $1999 ; 126$ : 1655-64.

34. Bendel-Stenzel MR, Gomperts M, Anderson R, et al. The role of cadherins during primordial germ cell migration and early gonad formation in the mouse. Mech Dev $2000 ; 91: 143-52$.

35. Williams DE, de Vries P, Namen AE, et al. The Steel factor. Dev Biol $1992 ; 151$ : 368 76.

36. Grant M, Zuccotti M, Monk M. Methylation of CpG sites of two X-linked genes coincides with $X$-inactivation in the female mouse embryo but not in the germ line. Nat Genet 1992 ; 2 : 161-6.
TIRÉS À PART

S. Viville 